November $\quad 9, \quad 2018 \quad 16: 57 \quad$ WSPC/INSTRUCTION $\quad$ FILE zychor proceedings'STORI08

International Journal of Modern Physics E

(C) World Scientific Publishing Company

\title{
EXCITED HYPERONS PRODUCED IN PROTON-PROTON COLLISIONS WITH ANKE AT COSY
}

\author{
IZABELLA ZYCHOR \\ Instytut Problemów Jadrowych, Pl-05-400 Świerk, Poland \\ i.zychor@fz-juelich.de \\ Received (received date) \\ Revised (revised date)
}

\begin{abstract}
Excited neutral hyperons $Y^{0 *}$ produced in the $p p \rightarrow p K^{+} Y^{0 *}$ reaction with a COSY beam momentum of $3.65 \mathrm{MeV} / \mathrm{c}$ have masses below $1540 \mathrm{MeV} / \mathrm{c}^{2}$. The ANKE spectrometer allows the simultaneous observation of different decay modes: $Y^{0 *} \rightarrow$ $\pi^{0} \Sigma^{0}, \pi^{\mp} \Sigma^{ \pm}, \pi^{0} \Lambda, K^{-} p$ by measuring kaons and pions of either charge in coincidence with protons.

We have found indications for a neutral excited hyperon resonance $Y^{0 *}$ with a mass of $M\left(Y^{0 *}\right)=(1480 \pm 15) \mathrm{MeV} / \mathrm{c}^{2}$ and a width of $\Gamma\left(Y^{0 *}\right)=(60 \pm 15) \mathrm{MeV} / \mathrm{c}^{2}$. The cross section for $Y^{0 *}$ is of the order of few hundred nanobarns. It can be either a $\Sigma^{0}$ or a $\Lambda$ hyperon and on the basis of existing data no conclusion could be made whether it is a three-quark baryon or an exotic state.

Missing- and invariant-mass techniques have been used to identify the $\Lambda(1405)$ resonance decaying via $\Sigma^{0} \pi^{0}$. The cross section for $\Lambda(1405)$ production is equal to $\left(4.5 \pm 0.9_{\text {stat }} \pm 1.8_{\text {syst }}\right) \mu \mathrm{b}$. The shape and position of the $\Lambda(1405)$ distribution are similar to those found from other decay modes, so no support is given to the two-pole model.
\end{abstract}

\section{Introduction}

The production and properties of hyperons have been studied for more than 50 years, mostly in pion and kaon induced reactions. Hyperon production in $p p$ collisions has been investigated close to threshold at SATURNE (Saclay, France) and COSY-Jülich. Reasonably complete information on $\Lambda(1116), \Sigma^{0}(1192), \Sigma^{0}(1385)$, $\Lambda(1405)$ and $\Lambda(1520)$ can be found in the Review of Particle Physics 1

For the $\Lambda(1405)$, in spite of rather high statistics achieved (the total world statistics is several thousand events), there are still open questions concerning the nature of this resonance: is it a singlet $q q q$ state in the frame of $\mathrm{SU}(3)$ or a quarkgluon $(u d s-q)$ hybrid, or a KN bound state? $2-10$

On the contrary, the $\Sigma(1480)$ hyperon is not well established yet and it is described as a 'bump' with unknown quantum numbers 1 The Crystal Ball experiment has not seen any indications for the resonance $\Sigma(1480)$ in the $\pi^{0} \Lambda$ invariant mass distribution measured in the reaction $K^{-} p \rightarrow \pi^{0} \pi^{0} \Lambda$, dominated by the $\Sigma(1385) ! 11$

The program to investigate hyperon production from $p p$ interactions at low energies is very well suited for the ANKE spectrometer operated at COSY-Jülich. 
November $\quad 9, \quad 2018 \quad 16: 57 \quad$ WSPC/INSTRUCTION $\quad$ FILE zychor proceedings'STORI08

2 I. Zychor

In Fig. 1 a simplified decay scheme of hyperons investigated in our experiments is presented.

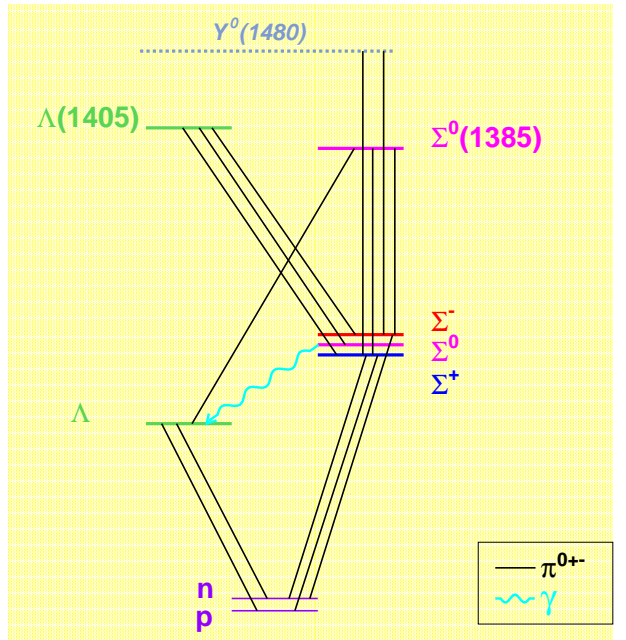

Fig. 1. Simplified decay scheme of investigated hyperons.

\section{Experiment and particle identification with ANKE}

The experiments have been performed with the ANKE spectrometer $\frac{12}{12}$ at the Cooler SYnchrotron COSY at the Research Center Jülich (Germany)! 13

COSY is a medium energy cooler synchrotron and storage ring for both polarized and unpolarized protons and deuterons. At COSY various targets can be used, e.g. solid or cluster-jet. COSY provides beams in the momentum range between 0.6 and $3.7 \mathrm{GeV} / \mathrm{c}$.

ANKE ("Apparatus for Studies of Nucleon and Kaon Ejectiles") is a magnetic spectrometer located at an internal target position of COSY. It consists of three dipole magnets, see Fig. 2. The central C-shaped spectrometer dipole D2, placed downstream of the target, separates the reaction products from the circulating COSY beam. The ANKE detection system, comprising range telescopes, scintillation counters and multi-wire proportional chambers, simultaneously registers both positively and negatively charged particles and measures their momenta. 14

The ANKE telescopes are used to register positively charged particles. They discriminate pions, kaons and protons with the same momenta due to their different energy losses. Passive copper degraders in the telescopes between the scintillation counters enhance the discrimination efficiency. The $K^{+}$mesons are stopped in the $\Delta E$ counters or in the second degrader of each telescope. Their decay, mainly into $\mu^{+} \nu_{\mu}$ and $\pi^{+} \pi^{0}$ with a lifetime of $\tau=12.4 \mathrm{~ns}$, provides a very effective criterion 
November $\quad 9, \quad 2018 \quad 16: 57 \quad$ WSPC/INSTRUCTION $\quad$ FILE zychor proceedings'STORI08

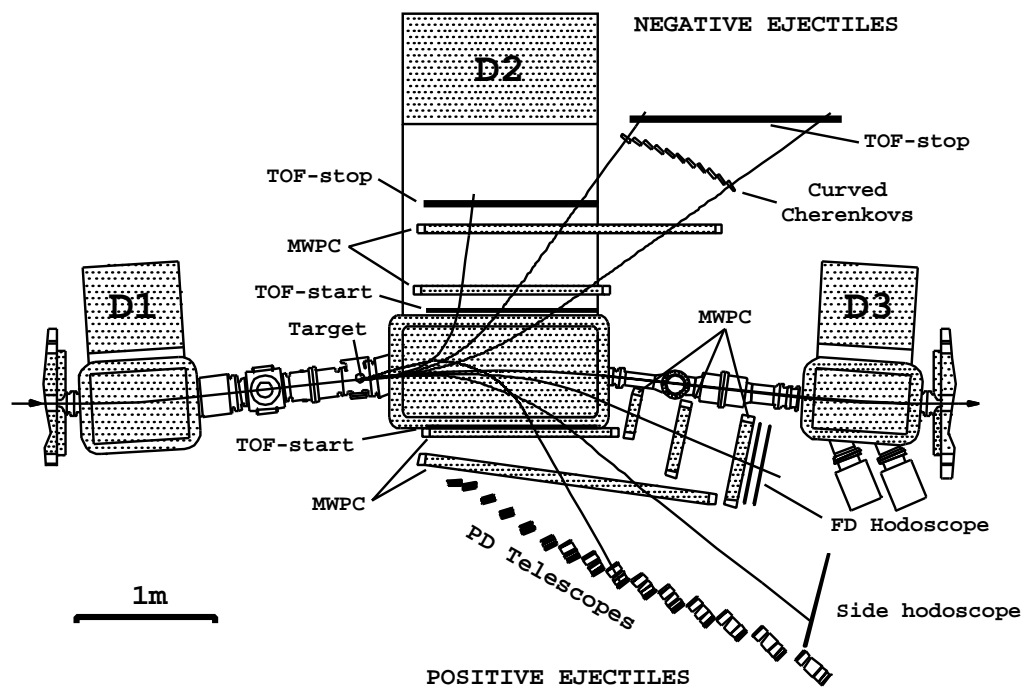

Fig. 2. ANKE spectrometer and detectors.

for kaon identification via detection of delayed signals in a so-called veto counter (with respect to prompt signals from e.g. a $\pi^{+}$produced in the target passing over all counters of a telescope). By measuring such delayed signals from the decay of stopped kaons, positively charged kaons can be identified at ANKE in a background of pions, protons and scattered particles up to $10^{6}$ times more intense ${ }^{15]}$ The use of veto counters causes a decrease of particle identification efficiency, typically by factor of 6 . The tracks of the ejectiles, measured with multi-wire proportional chambers (MWPCs), are used to reconstruct momenta of any registered particle.

Data originally taken for scalar meson $\frac{16}{16}$ and $\phi \frac{17}{}$ production have been used to study the production of low-lying hyperon resonances in $p p$ collisions with ANKE@COSY. Experiments have been performed in 2002 and 2005, respectively.

In Table 2 details of the experimental conditions are given.

Table 1. Experimental details.

\begin{tabular}{|c|c|c|}
\hline & $\begin{array}{l}4 \text { weeks in } 2002 \\
\text { for } \mathrm{Y}^{0 *}(1480)\end{array}$ & $\begin{array}{l}4 \text { weeks in } 2005 \\
\quad \text { for } \Lambda(1405)\end{array}$ \\
\hline integrated luminosity & $6 \mathrm{pb}^{-1}$ & $70 \mathrm{pb}^{-1}$ \\
\hline coincidences & 3 particles $\left(\mathrm{p}, \mathrm{K}^{+}, \pi^{+}\right.$or $\left.\pi^{-}\right)$ & 4 particles $\left(\mathrm{p}, \mathrm{p}, \mathrm{K}^{+}, \pi^{+}\right)$ \\
\hline $\begin{array}{l}K^{+} / \pi^{+} \text {momentum } \\
\text { p momentum }\end{array}$ & \multicolumn{2}{|c|}{$\begin{aligned} & 0.2-0.9 \mathrm{GeV} / \mathrm{c} \\
& >0.75 \mathrm{GeV} / \mathrm{c}\end{aligned}$} \\
\hline$\pi^{-}$momentum & $0.4-1.0 \mathrm{GeV} / \mathrm{c}$ & $0.2-1.0 \mathrm{GeV} / \mathrm{c}$ \\
\hline delayed veto for $K^{+}$ & yes & no \\
\hline detection efficiency & $7 \%$ & $55 \%$ \\
\hline mass resolution & $\sim 10 \mathrm{MeV} / \mathrm{c}^{2}$ & $\sim 20 \mathrm{MeV} / \mathrm{c}^{2}$ \\
\hline
\end{tabular}


November $\quad 9, \quad 2018 \quad 16: 57 \quad$ WSPC/INSTRUCTION $\quad$ FILE zychor proceedings'STORI08

4 I. Zychor

\section{Excited neutral hyperon $Y^{0 *}(1480)$}

Final states comprising a proton, a positively charged kaon, a pion of either charge and an unidentified residue $\mathrm{X}$ were investigated in the reaction $p p \rightarrow p K^{+} Y \rightarrow$ $p K^{+} \pi^{ \pm} X^{\mp}$ at the beam momentum of $3.65 \mathrm{GeV} / \mathrm{c}$. Kaons are identified by measuring delayed signals from the their decay, which, together with well-defined pions and protons, are used to determine the mass of $\mathrm{X}$.
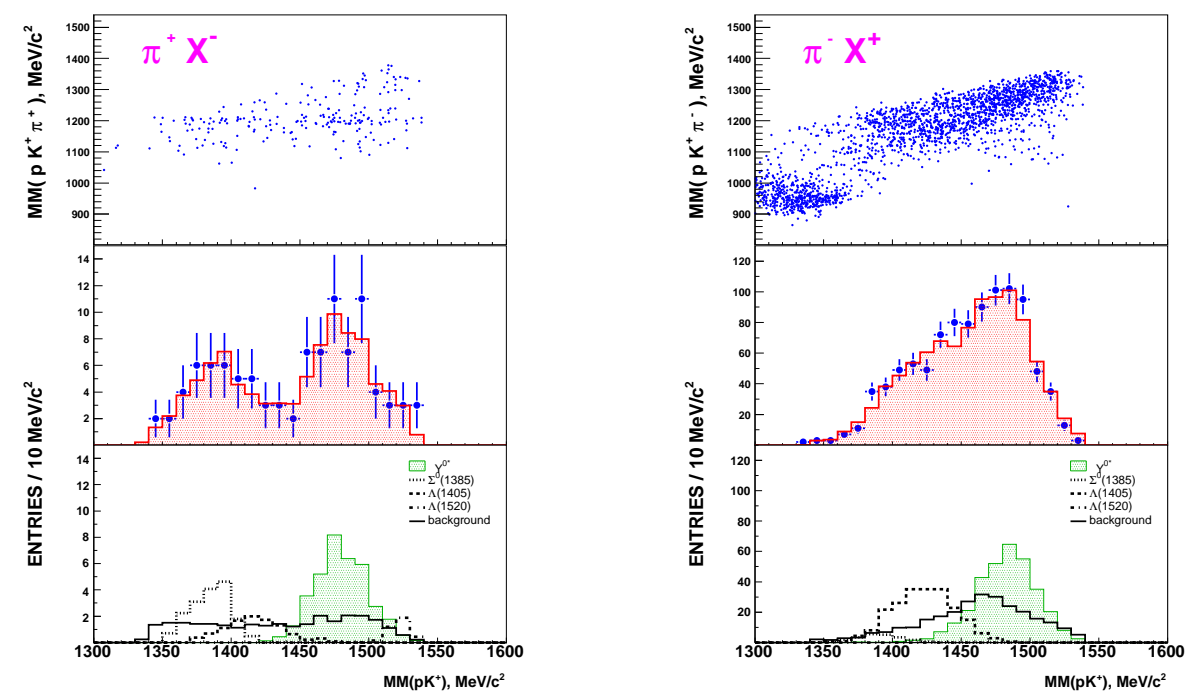

Fig. 3. Missing-mass $M M\left(p K^{+}\right)$spectra for the reaction $p p \rightarrow p K^{+} \pi^{+} X^{-}$(left) and $p p \rightarrow p K^{+} \pi^{-} X^{+}$(right). Upper parts: $M M\left(p K^{+}\right)$versus $M M\left(p K^{+} \pi\right)$ for $\pi^{+}$(right) and $\pi^{-}$ (left). Middle parts: Comparison of experimental (points) and simulated (shaded histograms) distributions. Lower parts: Non-resonant and resonant contributions to the overall simulated histograms.

In the upper part of Fig. 3 the missing mass distributions $M M\left(p K^{+} \pi\right)$ versus $M M\left(p K^{+}\right)$are shown for the reactions $p p \rightarrow p K^{+} \pi^{+} X^{-}$(left) and $p p \rightarrow p K^{+} \pi^{-} X^{+}$(right). Since the probability for detecting three-particle coincidences $\left(p K^{+} \pi^{+}\right)$is about an order of magnitude smaller than for $\left(p K^{+} \pi^{-}\right)$, the resulting numbers of events are also drastically different. In the distribution for the reaction $p p \rightarrow p K^{+} \pi^{+} X^{-}$(left) an enhancement corresponding to $X^{-}=\Sigma^{-}(1197)$, is observed on a low background. In the charge-mirrored $p p \rightarrow p K^{+} \pi^{-} X^{+}$case(right), the $\pi^{-}$may originate from different sources, e.g. a decay with the $\Sigma^{+}(1189)$ or a secondary decay of $\Lambda \rightarrow p \pi^{-}$, arising from the major background reaction $p p \rightarrow p K^{+} \Lambda \rightarrow p K^{+} \pi^{-} p$. Protons from this reaction are easily rejected by cutting the missing mass $M M\left(p K^{+} \pi^{-}\right)$around the proton mass. 
November $\quad 9, \quad 2018 \quad 16: 57 \quad$ WSPC/INSTRUCTION $\quad$ FILE zychor proceedings'STORI08

If only events around the $\Sigma$ mass are selected, then the missing mass spectrum $M M\left(p K^{+}\right)$in the reaction $p p \rightarrow p K^{+} \pi^{+} X^{-}$shows two peaks, see in the middleleft part in Fig. 3. One of them corresponds to the contribution of $\Sigma^{0}(1385)$ and $\Lambda(1405)$ hyperons. The second peak is located at a mass $\sim 1480 \mathrm{MeV} / \mathrm{c}^{2}$. In the $\pi^{-} X^{+}$case, the distribution also peaks at $1480 \mathrm{MeV} / \mathrm{c}^{2}$, see the right-middle part in Fig. 3 .

We have assumed that the measured missing mass $M M\left(p K^{+}\right)$spectra can be explained by the production of hyperon resonances and non-resonant contributions. Detailed Monte Carlo simulations have been performed including the production of well established excited hyperons $\left(\Sigma^{0}(1385), \Lambda(1405), \Lambda(1520)\right)$ and non-resonant contributions like $p p \rightarrow N K^{+} \pi X$ and $p p \rightarrow N K^{+} \pi \pi X ; X$ denotes any hyperon which could be produced in the experiment. For both final states the shape of the measured distributions cannot be reproduced by the simulations and an excess of events is observed around the missing mass of $1480 \mathrm{MeV} / \mathrm{c}^{2}$. Thus, an the excited hyperon $Y^{0 *}$ decaying via $\pi^{ \pm} X^{\mp}$ was included into simulations. The best fit to the experimental data was obtained for the $Y^{0 *}$ with a mass $M\left(Y^{0 *}\right)=(1480 \pm$ 15) $\mathrm{MeV} / \mathrm{c}^{2}$ and a width $\Gamma\left(Y^{0 *}\right)=(60 \pm 15) \mathrm{MeV} / \mathrm{c}^{2}$, see lower parts in Fig. 3. There have been identified 100 and 1000 events for $\pi^{+} X^{-}$and $\pi^{-} X^{+}$case, respectively. The statistical significance of the signal, assuming that this is due to the production of the $Y^{0 *}$, is between 4 and $6 \sigma$ depending on a procedure. The production cross section is of the order of few hundred nanobarns 18

\section{The $\Lambda(1405)$ hyperon}

The $p p \rightarrow p K^{+} p \pi^{-} X^{0}$ reaction is selected by a multiparticle final state, containing two protons, a positively charged kaon, a negatively charged pion and an unidentified residue $X^{0}$. In the $\Sigma^{0}(1385) \rightarrow \Lambda \pi^{0}$ decay the $X^{0}$ residue is a $\pi^{0}$ while, for the $\Lambda(1405) \rightarrow \Sigma^{0} \pi^{0}$ decay, $X^{0}=\pi^{0} \gamma$ (see Fig. 1). In the upper part of Fig. 4 a distribution of $M M\left(p_{F d} K^{+}\right)$versus $M M\left(p K^{+} \pi^{-} p\right)$ is plotted for events with the invariant mass $M\left(p_{S d} \pi^{-}\right)$of the $p_{S d} \pi^{-}$pairs corresponding to the mass of the $\Lambda$, i.e. between 1112 and $1120 \mathrm{MeV} / \mathrm{c}^{2}$. The two horizontal bands show the four-particle missing-mass $M M\left(p K^{+} \pi^{-} p\right)$ criteria used to separate the $\Sigma^{0}(1385)$ candidates from those of the $\Lambda(1405)$. The lower band is optimised to identify a $\pi^{0}$ whereas the upper one selects masses significantly greater than $m\left(\pi^{0}\right)$.

In order to extract the $\Lambda(1405)$ distribution from the measured $\Sigma^{0} \pi^{0}$ decay, the non-resonant contributions have been fitted to the experimental data. After subtracting them from the data, the distribution shown as experimental points in the lower panel of Fig. 4 was obtained. 156 events have been identified in this spectrum.19

The $(\Sigma \pi)^{0}$ invariant-mass distributions have been previously studied in two hydrogen bubble chamber experiments. Thomas et al. found $\sim 400 \Sigma^{+} \pi^{-}$or $\Sigma^{-} \pi^{+}$ events corresponding to the $\pi^{-} p \rightarrow K^{0} \Lambda(1405) \rightarrow K^{0}(\Sigma \pi)^{0}$ reaction at a beam momentum of $1.69 \mathrm{GeV} / \mathrm{c}$. ${ }^{20}$ Hemingway used a $4.2 \mathrm{GeV} / \mathrm{c}$ kaon beam to investi- 


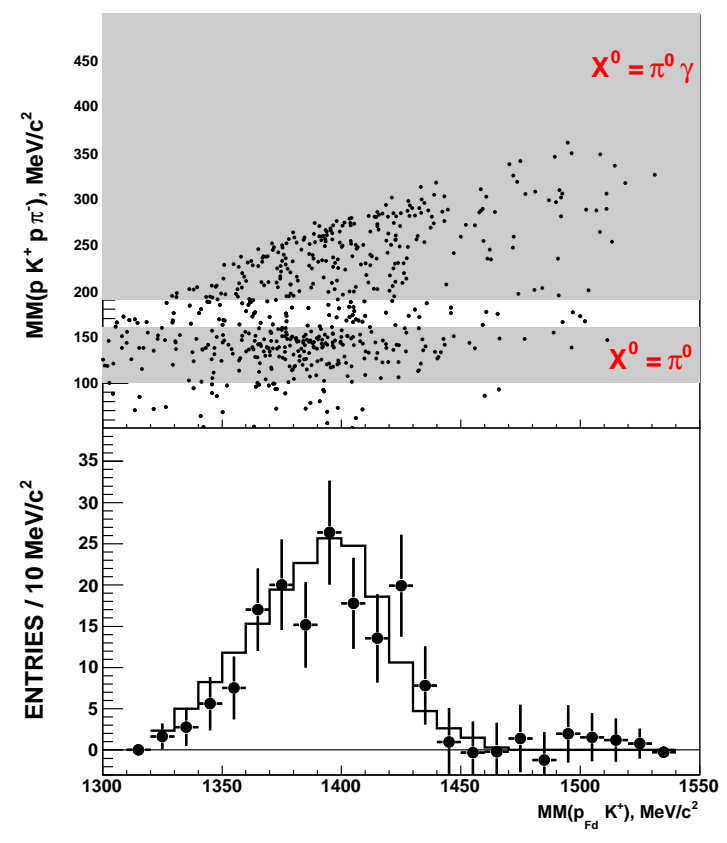

Fig. 4. Upper panel: Missing mass $M M\left(p K^{+} \pi^{-} p\right)$ versus $M M\left(p_{F d} K^{+}\right)$with the shaded horizontal boxes showing the $M M\left(p K^{+} \pi^{-} p\right)$ bands used for event selection. The lower one is located around the $\pi^{0}$ mass and the upper one selects $M M\left(p K^{+} \pi^{-} p\right)>190 \mathrm{MeV} / c^{2}$, significantly greater than the $\pi^{0}$ mass. Lower panel: The background-subtracted line shape of the $\Lambda(1405)$ decaying into $\Sigma^{0} \pi^{0}$ (points) compared to the spectrum of the $\Lambda$ (1405) from the LEPS experiment for photon energy range of $1.5<E_{\gamma}<2.0 \mathrm{GeV}$ (solid line).

gate $K^{-} p \rightarrow \Sigma^{+}(1660) \pi^{-} \rightarrow \Lambda(1405) \pi^{+} \pi^{-} \rightarrow\left(\Sigma^{+} \pi^{-}\right) \pi^{+} \pi^{-}$and measured 1106 events. ${ }^{21}$ Recently, the LEPS experiment has investigated the $\Lambda(1405)$ hyperon production in the $\gamma p \rightarrow K^{+} Y^{*}$ reaction. ${ }^{22}$ The $\Lambda(1405)$ hyperon was measured in the $\left(K^{+} \Sigma^{ \pm} \pi^{\mp}\right)$ final state, where the contamination from $\Sigma^{0}(1385)$ was estimated from the $\left(K^{+} \Lambda \pi^{0}\right)$ final state. In the lower panel in Fig 4 our experimental points are compared to the results of the LEPS experiment (for comparison with data of Thomas and Hemingway see Ref. 19). Despite the very different production mechanisms, all four distributions have similar shapes and positions. This might suggest that, if there are two states present in this region, then the reaction mechanisms in the four cases are preferentially populating the same one. It should, however, be noted that by identifying a particular reaction mechanism, the proponents of the two-state solution can describe the shape of the distribution that we have found 23 
November $\quad 9, \quad 2018 \quad 16: 57 \quad$ WSPC/INSTRUCTION $\quad$ FILE zychor proceedings'STORI08

Excited Hyperons Produced in pp Collisions with ANKE@COSY

\section{Outlook}

The decay of excited hyperons $Y^{0 *}$ via $\Lambda \pi^{0}$ and $\Sigma^{0} \pi^{0} \rightarrow \Lambda \gamma \pi^{0}$ can be detected directly in electromagnetic calorimeters by registering neutral particles, i.e. $\gamma$ and/or $\pi^{0}$. Measurements of such channels are discussed for $\gamma p$ reactions with $\mathrm{CB} / \mathrm{TAPS}$ at ELSA ${ }^{24}$ and are also planned in $p p$ collisions with WASA at COSY 25

\section{Acknowledgements}

We thank all other members of the ANKE collaboration and the COSY accelerator staff for their help during the data taking. This work has been supported by COSYFFE Grant, BMBF, DFG and Russian Academy of Sciences.

\section{References}

1. C. Amsler et al. (Particle Data Group), Phys. Lett. B 667 (2008) 1.

2. U. Löring, B.C. Metsch and H.R. Petry, Eur. Phys. J. A 10 (2001) 395.

3. W. Melnitchouk et al., Phys. Rev. D 67 (2003) 114506.

4. R. Arnold and J. Sakurai, Phys. Rev. 128 (1962) 2808.

5. R. Dalitz, T. Wong and G. Rajasekaran, Phys. Rev. 153 (1967) 1617.

6. M. Jones, R.H. Dalitz and R.R. Horgan, Nucl. Phys. B 129 (1977) 45.

7. E. Oset and A. Ramos, Nucl. Phys. A 635 (1998) 99).

8. J.A. Oller and U.G. Meissner, Phys. Lett. B 500 (2001) 263.

9. E. Oset, A. Ramos and C. Bennhold, Phys. Lett. B 527 (2002) 99 [Erratum-ibid. B 530 (2002) 260].

10. D. Jido et al., Nucl. Phys. A 725 (2003) 181.

11. S. Prakhov et al., Phys. Rev. C 69 (2004) 042202(R).

12. S. Barsov et al., Nucl. Instr. Methods Phys. Res., Sect. A 462 (2001) 364.

13. R. Maier, Nucl. Instr. Methods Phys. Res., Sect. A 390 (1997) 1.

14. M. Büscher et al., Nucl. Instr. Methods Phys. Res., Sect. A 481 (2002) 378.

15. M. Büscher et al., Eur. Phys. J. A 22 (2004) 301.

16. M. Büscher et al., COSY proposal \#55 (2001), www.fz-juelich.de/ikp/anke/.

17. M. Hartmann, et al., COSY proposal \#204 (2002), www.fz-juelich.de/ikp/anke/.

18. I. Zychor et al., Phys. Rev. Lett. 96 (2006) 012002.

19. I. Zychor et al., Phys. Lett. B 6660 (2008) 167.

20. D.W. Thomas et al., Nucl. Phys. B 56 (1973) 15.

21. R.J. Hemingway, Nucl. Phys. B 253 (1984) 742.

22. M. Niiyama et al., Phys. Rev. C 78 (2008) 035202.

23. L.S. Geng and E. Oset, Eur. Phys. J. A 34 (2007) 405.

24. H. Schmieden, Letter of Intent ELSA/4-2003.

25. H.-H. Adam et al., nucl-ex/0411038. 